\title{
Association between conventional risk factors and different ultrasound-based markers of atherosclerosis at carotid and femoral levels in a middle-aged population
}

\author{
Patrick Yerly • Nicolas Rodondi - Barathi Viswanathan • \\ Walter Riesen · Pierre Vogt $\cdot$ Pascal Bovet
}

Received: 23 March 2012/Accepted: 3 September 2012/Published online: 27 September 2012

(C) Springer Science+Business Media, B.V. 2012

\begin{abstract}
Ultrasound detection of sub-clinical atherosclerosis (ATS) may help identify individuals at high cardiovascular risk. Most studies evaluated intima-media thickness (IMT) at carotid level. We compared the relationships between main cardiovascular risk factors (CVRF) and five indicators of ATS (IMT, mean and maximal plaque thickness, mean and maximal plaque area) at both carotid and femoral levels. Ultrasound was performed on 496 participants aged 45-64 years randomly selected from the general population of the Republic of Seychelles. $73.4 \%$ participants had $\geq 1$ plaque (IMT thickening $\geq 1.2 \mathrm{~mm}$ ) at carotid level and $67.5 \%$ at femoral level. Variance (adjusted R2) contributed by age, sex and CVRF (smoking, LDL-cholesterol, HDL-cholesterol, blood pressure, diabetes) in predicting any of the ATS markers was larger at femoral than carotid level. At both carotid and femoral levels, the association between CVRF and ATS
\end{abstract}

P. Yerly $(\bowtie) \cdot$ P. Vogt

Department of Cardiology, Lausanne University Hospital

(CHUV), Rue du Bugnon 46, 1011 Lausanne, Switzerland

e-mail: patrick.yerly@chuv.ch

P. Yerly $\cdot$ B. Viswanathan $\cdot$ P. Bovet

Unit for Prevention and Control of Cardiovascular Disease,

Ministry of Health, Victoria, Republic of Seychelles

N. Rodondi

Department of Ambulatory Care and Community Medicine,

Lausanne University Hospital, Lausanne, Switzerland

W. Riesen

Institute of Clinical Chemistry and Hematology,

Canton Hospital, St-Gallen, Switzerland

P. Bovet

Institute of Social and Preventive Medicine (IUMSP),

Lausanne University Hospital, Lausanne, Switzerland was stronger based on plaque-based markers than IMT. Our findings show that the associations between CVRF and ATS markers were stronger at femoral than carotid level, and with plaque-based markers rather than IMT. Pending comparison of these markers using harder cardiovascular endpoints, our findings suggest that markers based on plaque morphology assessed at femoral artery level might be useful cardiovascular risk predictors.

Keywords Atherosclerosis - Ultrasonography · Carotid artery $\cdot$ Femoral artery $\cdot$ Intima-media thickness $\cdot$ Plaque . Risk factors

\section{Introduction}

Ultrasound screening for pre-symptomatic peripheral atherosclerosis (ATS) is increasingly recognized as a useful method for enhancing the detection of high risk subjects beyond cardiovascular risk factors (CVRF) [1-4]. So far, the assessment of peripheral ATS mainly relied on the measurement of the intima-media thickness (IMT) on predetermined wall segments of the carotid arteries. Carotid IMT can be considered both as a surrogate marker of generalized ATS [5] and an independent predictor of cardiovascular events and mortality [1-3, 6-9]. However, several limitations have prevented a broad implementation of this technique into clinical practice, e.g. the lack of standardized reference cut-off values [10] and the influence of factors not related to ATS affecting media thickness $[11,12]$. Moreover, concerns have been raised regarding the small added contribution of carotid IMT to the estimation of an individual's risk of cardiovascular events as compared to CVRF alone [13]. 
In order to identify possibly better vascular outcomes, several authors have focused on alternate markers like the detection of ATS plaques. The presence and burden of plaques have been associated with incident stroke or myocardial infarction and cardiovascular mortality [1, 14-25], but methods for quantifying plaques have largely differed between studies. Given decreased statistical power associated with the use of dichotomized variables (i.e. plaque vs. no plaque), investigators have focused on continuous quantitative variables, such as plaque thickness [25] and plaque area [18, 21, 26, 27]. Furthermore, the sites for the detection of plaques have also varied. Although carotid arteries have been examined most often [1, 14, 17-19, 21, 22, 24-27], a few studies have assessed the significance of ATS markers at the femoral artery level [15, 16, 20, 23]. To our best knowledge, the predictive value of plaque area has not been evaluated at femoral artery level.

Hence, several ultrasound-based ATS markers have been used and their comparative predictive values are yet unclear. Large prospective studies will be needed to compare the predictive significance of these different markers. Pending such costly and years-long longitudinal studies, useful information can be suggested by comparing the associations of CVRF with candidate ATS markers at both carotid and femoral levels.

In this study, we compared the associations between five ATS markers (mean IMT, mean plaque thickness, maximal plaque thickness, mean plaque area and maximal plaque area) and major conventional CVRF in a population-based sample of middle-aged adults. Furthermore, we compared these relationships at both the carotid and femoral artery levels.

\section{Methods}

\section{Study population}

This study was conducted in the Republic of Seychelles. The country is an archipelago of 115 islands located approximately $1,800 \mathrm{~km}$ east of Kenya in the Indian Ocean (African region). About $99 \%$ of the inhabitants live on the largest three islands and the majority of the population is of African descent. During the last 2-3 decades, the country has experienced fast socio-economic growth, largely driven by booming tourism and fishing industries, and the gross national product per capita currently exceeds US \$8000. CVD accounts for approximately $38 \%$ of total mortality and life expectancy is 69 years in men and 76 years in women [28]. A high prevalence of several major risk factors was found in 1989 and 2004, e.g. high blood pressure, overweight, and diabetes [29-31]. Consistent with fairly high levels of risk factors in the population, a previous study in 1994 showed a high prevalence of peripheral atherosclerosis (plaques) in the adult population [32].

The design, methods and overall results of this population-based study have been reported elsewhere [30, 33]. Briefly, a random sex- and age-stratified sample of all inhabitants aged 25-64 was drawn using computerized data of a national population census carried out in 2002 and thereafter regularly updated by civil status authorities. From the initial sample of 1,632 individuals, 69 were dead, abroad or could not be traced and $80.1 \%$ of the remaining eligible subjects attended the survey. The study was approved by the Ministry of Health after technical and ethical reviews and all participants gave written informed consent.

\section{Study sample}

Participants in this study included all 501 consecutive participants (225 men and 276 women) aged 45-64 who attended the study during the first 17 weeks. We restricted ultrasound to this age range because middle-aged individuals are more likely to present ATS plaques than younger persons. Restriction to a 17 -week period corresponded to the period during which an experienced ultrasonographer (PY) was available. We excluded 5 subjects for whom femoral ultrasound could not be performed in good conditions because of excessively deep location of the artery $(>4 \mathrm{~cm})$. The total sample for this study includes 496 subjects (223 men and 273 women).

\section{Cardiovascular risk factors}

A team of nurses with previous experience in conducting surveys administered a structured questionnaire and performed a physical examination. Smoking status was defined as current smoking of at least 1 cigarette per day. Blood pressure was defined as the average of the last two of three measurements taken with a mercury sphygmomanometer at intervals of at least 2 min after the participants had been seating for at least $30 \mathrm{~min}$ and using a cuff size appropriate for the arm circumference.

Venous blood was drawn in the morning after an overnight fast. All samples were centrifuged within $2 \mathrm{~h}$ of blood collection and plasma was immediately frozen to $-20{ }^{\circ} \mathrm{C}$. Total blood cholesterol, high density lipoprotein (HDL)-cholesterol and triglycerides were measured by standard enzymatic colorimetric tests (CHOD-PAP, HDLC plus 2nd generation, GPO-PAP, Roche Diagnostics) on a Hitachi 917 analyzer (Roche Diagnostics). Low density lipoprotein (LDL)-cholesterol was calculated using the Friedwald formula.

Fasting glucose was determined on venous blood with a point-of-care analyzer (Cholestec LDX, Hayward, USA). 
If the value was $\geq 5.6 \mathrm{mmol} / \mathrm{l}$, an additional capillary measurement -adjusted for plasma values- was carried out (Ascencia Elite glucometer, Bayer) and the average of the two readings was considered. If blood glucose was $\geq 5.6-7.0 \mathrm{mmol} / \mathrm{l}$, an oral 75 -gram glucose tolerance test was performed. Diabetes mellitus was defined as fasting blood glucose $\geq 7 \mathrm{mmol} / \mathrm{l}$, a 2 -h post-load glucose $\geq 11.1 \mathrm{mmol} / \mathrm{l}$, or current hypoglycemic medication [34].

\section{Ultrasonography}

High resolution B-mode ultrasound was performed with a portable system (LOGIQ Book, general electric health care, Waukesha, USA) connected to a $6-10 \mathrm{MHz}$ linear array transducer. The ultrasound system was equipped with a software of arterial wall analysis with a semi-automated edge detection system and calibrable electronic caliper (M'ATH, ICN-Metris, Paris, France). All scans and measurements were performed by the same experienced investigator (PY), who was blinded to the CVRF status of the subjects. All measurements were performed directly on frame.

Four vascular sites were examined in all participants: the right and left carotid and femoral arteries. Carotid arteries were examined from the supraclavicular fossa to the submandibular angle, including the common carotid artery (CCA), the bulb, and the origin of the internal and external carotid arteries. Femoral arteries were examined from $4 \mathrm{~cm}$ above the bifurcation spur in their common portion (CFA) to $4 \mathrm{~cm}$ in their superficial branch and at the origin of their profound branch. According to recent consensus recommendations [35], all scans were acquired at a start depth of $3 \mathrm{~cm}$ with a $10 \mathrm{MHz}$ probe frequency, allowing the best image resolution on screen. These parameters could be adjusted to a maximum of $4 \mathrm{~cm}$ for depth and $8 \mathrm{MHz}$ for probe frequency depending on individual anatomic considerations.

\section{IMT protocol}

Intima-media thickness, defined as the distance from the leading edge of the lumen-intima interface to the leading edge of the media-adventitia interface, was measured on the far wall of both CCAs and CFAs on a $10 \mathrm{~mm}$ segment located $2 \mathrm{~cm}$ upstream from the flow divider. Optimal longitudinal frames of these segments were frozen in late diastole before analysis with the M'ATH software. The recorded IMT value was the mean thickness measured along the whole segment. In case of plaque occurrence on the reference site, the mean IMT value was substituted for the maximal thickness of this plaque. Finally, the mean values on each side were averaged to obtain a single mean variable at the carotid and femoral levels and a combined value for all the four sites.

\section{Plaques and ATS burden protocol}

ATS plaques were defined as a focal wall thickening $\geq 1,200 \mu \mathrm{m}$ protruding into the arterial lumen [36, 37]. Plaques were identified on both the near and the far walls in all the above described arterial segments by thorough transversal and longitudinal scanning. After detection, plaques were scanned in the best longitudinal view perpendicularly crossing the most prominent part of the lesions, and scans frozen in late diastole. The ATS burden was quantified by measuring two parameters, plaque thickness and plaque area.

Plaque thickness was considered as the distance between the plaque-lumen interface and the plaque-adventitia interface of the thickest plaque visualized on each site. We defined "mean maximal thickness" as the average of the maximal plaque thickness values measured on both left and right sides at carotid, femoral and overall levels, respectively. If there was no plaque in one of the considered sites, plaque thickness was substituted by the IMT value. We defined "maximal thickness" as the maximal plaque thickness observed on either the left or right sides at carotid, femoral and overall levels, respectively. In the absence of plaque, maximal plaque thickness was replaced by the IMT value.

Plaque area was measured for all visible plaques identified in any of the four defined artery sites. Surface measurement was based on longitudinal views showing the largest extent of each identified plaque. The perimeter of each identified plaque was outlined on frame by means of an electronic cursor and plaque area was automatically calculated by the software $[18,26]$. Maximal plaque area refers to the largest total plaque surface found on either left or right side at the carotid, femoral or overall levels, respectively. Mean plaque area corresponds to the mean of the total area of all plaques found on both left and right arteries at carotid, femoral or overall levels, respectively.

\section{Reproducibility}

The intra-observer reproducibility was achieved by a second evaluation of 40 vascular sites on 10 randomly selected participants at a time interval of 26-60 days between the two examinations. The variability for the presence of plaques showed a $95 \%$ agreement between measurements at the first and second examinations with a Kappa value of 0.9 $(P<0.001)$. For carotid IMT, the coefficient of variation was $4.8 \%$, which is similar to previous studies on IMT reproducibility [38]. For femoral IMT, the coefficient was $9.2 \%$. For maximal plaque thickness, the coefficient of variation was 9.5 and $6.8 \%$ at carotid and femoral levels, 
respectively. For maximal plaque area, coefficients were 18.8 and $13.9 \%$ at carotid and femoral levels, respectively.

\section{Statistical analysis}

The distribution of the different ATS markers was tabulated by sex and age. We used multivariate linear regression to analyze the independent association between risk factors and each of the five ATS markers. In order to facilitate the comparison of various models with different ATS markers, we displayed the standardized regression coefficients, which represent the change in the dependent variables resulting from a change of one standard deviation in each of the independent variables. We then calculated the variance ( $\mathrm{R}$ squared values) of the multivariate models, i.e. the proportion of variability in the data set that is accounted for by the independent variables. We displayed the R2 contributed by all variables (full models) and by models adjusted only for age and sex. The difference in variance between the full model and the model with only age and sex corresponds to the proportion of variability in predicting the ATS markers that is contributed by the modifiable risk factors, independent of age and sex. Pearson correlation coefficients were calculated both between different ATS markers and between same makers at carotid and femoral levels. Significance was defined for two-sided $P$ values less than 0.05 . Analyses were performed with Stata 8.2.

\section{Results}

Clinical, biochemical and ultrasonographic characteristics of the participants are shown in Table 1. ATS plaques were detected in the carotid arteries of 368 participants (73.4\%), of whom 245 had plaques $\geq 1,500 \mu \mathrm{m}(49.4 \%$ of all participants). In the femoral arteries, plaques were detected in 335 subjects $(67.5 \%)$, of whom 274 had plaques $\geq 1,500 \mu \mathrm{m}$ (55.2\% of all participants). Figure 1 shows the distribution of plaques identified in any of the four considered carotid or femoral locations according to age and sex. Men had plaques on more artery sites than women. The proportion of men and women with at least 1 plaque on $\geq 1$ artery site increased sharply with age. At carotid artery level, uni/ bilateral plaques were located on the site for IMT measurement in only $18 / 5$ subjects (of a total of 368 individuals with carotid plaques) but in as many as 102/67 subjects at femoral artery level (of a total of 335 individuals with femoral plaques). Hence, IMT was measured independently of plaques in most instances at carotid level while IMT represents the thickness of a plaque in approximately a quarter of subjects at femoral level. Table 2 shows the mean and maximal values of the considered ATS markers by sex and age. Mean and maximal ATS markers were larger in men than in women and these values increased significantly with age (e.g. confidence intervals [mean \pm 1.96 $\mathrm{SE}]$ did not overlap in younger and older age categories for all markers in either gender). The proportional increase over age was smaller for IMT (up to $50 \%$ increase) than for thickness and area (up to $400 \%$ ). The relative increase in size of the ATS markers over age was of a similar order of magnitude in carotid and femoral arteries.

Age was generally the strongest predictor for all ATS markers in a model of age and sex adjusted multivariate analysis between risk factors and ATS markers (Table 3). All risk factors (LDL-cholesterol, HDL-cholesterol, systolic blood pressure, smoking, diabetes) were strongly associated with all ATS markers (IMT, thickness and area) at femoral level, but only LDL cholesterol and blood pressure were consistently associated with the ATS markers at carotid level. For most modifiable risk factors, the strength of the association (i.e. the magnitude of the regression coefficient) was higher at femoral than carotid level. Models based on both the carotid and femoral arteries did not show stronger associations with CVRF than models based on the femoral arteries alone. Table 4 shows the variance (adjusted R2) for predicting each of the four ATS markers in three different models adjusting for 1)age, sex and all considered modifiable risk factors (LDL-cholesterol, HDL-cholesterol, systolic blood pressure, smoking and diabetes mellitus), 2) age and sex only, and 3) modifiable risk factors only (i.e. variance explained by the full model minus variance explained by age and sex only). Several observations can be done. First, variance contributed by age, sex and risk factors (full model) was larger at femoral than carotid level, and the larger variance at femoral level tended to be contributed by both modifiable risk factors and age/sex. Second, variance contributed by risk factors and age/sex tended to be larger for plaque-based markers than for IMT. Third, variance tended to be larger when models were based on both carotid and femoral arteries vs. either carotid or femoral site for markers based on mean estimates (as opposed to models based on maximal plaque thickness or area). Fourth, modifiable risk factors tended to account for more variance than age among men while age tended to contribute more variance in women, particularly at femoral level. This may be consistent with lower prevalence of plaques and more favorable levels of risk factors in middleaged women than in men.

Finally, all plaque-based markers were highly correlated with each other (correlation coefficient 0.73-0.97) at both carotid and femoral levels in men and women (Table 5), but much less with IMT. This suggests that IMT and plaque-based markers may convey different information. Conversely, the generally high correlation coefficients 
Table 1 Characteristics of the participants

\begin{tabular}{|c|c|c|c|c|}
\hline & \multicolumn{2}{|l|}{$\operatorname{Men}(n=223)$} & \multicolumn{2}{|c|}{ Women $(n=273)$} \\
\hline & Mean/percent & SD & Mean/percent & SD \\
\hline Age (years) & 54.0 & 5.8 & 54.1 & 6.0 \\
\hline Systolic blood pressure $(\mathrm{mmHg})$ & 139.9 & 20.1 & $135.7 * *$ & 20.6 \\
\hline Diastolic blood pressure $(\mathrm{mmHg})$ & 90.2 & 11.9 & $85.9 * *$ & 12.1 \\
\hline Total cholesterol (mmol/l) & 5.6 & 1.2 & $5.9 * *$ & 1.4 \\
\hline LDL-cholesterol $(\mathrm{mmol} / \mathrm{l})$ & 3.6 & 1.2 & $4.0 * * *$ & 1.3 \\
\hline HDL-cholesterol $(\mathrm{mmol} / \mathrm{l})$ & 1.4 & 0.5 & 1.4 & 0.4 \\
\hline Body mass index & 26.4 & 5.1 & $30.0 * * *$ & 6.0 \\
\hline Current smoking (\%) & 30.0 & & $3.6 * * *$ & \\
\hline Blood pressure $\geq 140 / 90 \mathrm{mmHg}(\%)$ & 58.3 & & $45.8 * *$ & \\
\hline Blood pressure $\geq 140 / 90 \mathrm{mmHg}$ or treatment $(\%)$ & 65.5 & & 63.4 & \\
\hline Overweight $\left(\mathrm{BMI} \geq 25 \mathrm{~kg} / \mathrm{m}^{2}\right)(\%)$ & 59.2 & & $81.0 * * *$ & \\
\hline Obesity (BMI $\left.\geq 30 \mathrm{~kg} / \mathrm{m}^{2}\right)(\%)$ & 19.7 & & $44.3^{* * *}$ & \\
\hline Diabetes $(\%)$ & 17.5 & & $21.2 *$ & \\
\hline
\end{tabular}

Diabetes fasting blood glucose $\geq 7 \mathrm{mmol} / \mathrm{l}$ or history of treatment for diabetes $S D$ standard deviation $P$ value for the comparison between men and women $* P<0.05$, ** $P<0.01$, *** $P<0.001$
IMT. This study provides a first head-to-head comparison of IMT, plaque thickness and plaque area at both carotid and femoral levels, and this is the first study to examine the "plaque thickness" parameter in the femoral artery.

Our findings suggest that the measurements of ATS markers at the femoral level could better reflect the exposure to risk factors than measurements at the carotid level. Only few reports have examined the relationship between peripheral ATS at both carotid and femoral artery levels and cardiovascular risk. Although our findings are based on the proxy measurement of CVRF and not on hard CVD outcomes like myocardial infarction, stroke or CV death, it is still interesting to relate them with studies that have correlated ATS markers with incident CVD outcomes. In a prospective study including 10,000 subjects, Belcaro et al. found that the incidence of CVD events was associated with pre-symptomatic peripheral ATS, and that combining carotid and femoral findings provided incremental prediction as compared to findings at the sole carotid level, but with no difference between both vascular fields taken separately [15]. The apparent discrepancy with our results (larger association between ATS markers and CVRF at femoral than carotid levels in our study) can be explained by different factors. First, Belcaro et al. included only very low-risk subjects in their study (excluding those with diabetes mellitus, total cholesterol $>5.2 \mathrm{mmol} / \mathrm{l}$ and/or hypertension) who are less likely to develop significant plaques at any artery level (normal arteries in $79.9 \%$ of their participants vs. $10.5 \%$ in our study). Second, markers of CVD risk factors may differ from markers of CVD events. However, our results concur with findings by Belcaro in the sense that combining carotid with femoral findings might increase the value of ATS markers. In another prospective study on high risk subjects with stable angina pectoris (558 patients, median follow-up 3.0 years),
In this general population of middle-aged adults, ATS markers were more strongly associated with CVD risk factors at femoral than carotid artery level and associations tended to be stronger with plaque-based markers than with 
Table 2 Mean values of different markers of peripheral atherosclerosis by sex and age

\begin{tabular}{|c|c|c|c|c|c|c|c|c|c|c|c|c|c|}
\hline & & \multicolumn{6}{|l|}{ Men } & \multicolumn{6}{|l|}{ Women } \\
\hline & & \multicolumn{2}{|c|}{ Carotid } & \multicolumn{2}{|c|}{ Femoral } & \multicolumn{2}{|c|}{ Combined } & \multicolumn{2}{|l|}{ Carotid } & \multicolumn{2}{|l|}{ Femoral } & \multicolumn{2}{|c|}{ Combined } \\
\hline & & Mean & SE & Mean & SE & Mean & $\mathrm{SE}$ & Mean & SE & Mean & SE & Mean & SE \\
\hline \multirow[t]{4}{*}{ Mean IMT $(\mu \mathrm{m})$} & $45-49$ & 712 & 18 & 953 & 70 & 831 & 78 & 679 & 8 & $678 * * *$ & 33 & $678 * * *$ & 17 \\
\hline & $50-59$ & 726 & 13 & 1172 & 109 & 949 & 112 & 736 & 20 & $829 * *$ & 68 & $782 * *$ & 38 \\
\hline & $55-59$ & 760 & 19 & 1143 & 89 & 952 & 95 & 799 & 29 & 937 & 47 & 869 & 30 \\
\hline & $60-65$ & 793 & 16 & 1415 & 129 & 1104 & 136 & 880 & 32 & 1257 & 67 & 1069 & 46 \\
\hline \multirow[t]{4}{*}{ Mean plaque thickness $(\mu \mathrm{m})$} & $45-49$ & 1035 & 53 & 1232 & 84 & 1133 & 52 & 986 & 44 & $810 * * *$ & 44 & $898 * *$ & 33 \\
\hline & $50-59$ & 1270 & 79 & 1555 & 110 & 1413 & 73 & 1127 & 46 & $1197 * *$ & 89 & $1162 * * *$ & 52 \\
\hline & $55-59$ & 1377 & 78 & 1678 & 117 & 1527 & 81 & 1292 & 62 & $1255^{*}$ & 61 & $1274^{*}$ & 45 \\
\hline & $60-65$ & 1853 & 107 & 1906 & 131 & 1879 & 93 & $1489 * * *$ & 72 & $1637 *$ & 83 & $1563 * * *$ & 63 \\
\hline \multirow[t]{4}{*}{ Maximal plaque thickness $(\mu \mathrm{m})$} & $45-49$ & 1282 & 81 & 1454 & 101 & 1759 & 92 & 1223 & 97 & $1002 * * *$ & 66 & $1424 * * *$ & 71 \\
\hline & $50-59$ & 1503 & 97 & 1885 & 129 & 2176 & 113 & 1368 & 93 & $1482 *$ & 99 & $1741 * *$ & 81 \\
\hline & $55-59$ & 1718 & 102 & 2062 & 132 & 2340 & 109 & 1591 & 125 & $1567 *$ & 82 & 1956 & 81 \\
\hline & $60-65$ & 2218 & 139 & 2248 & 144 & 2738 & 140 & $1747 * *$ & 144 & $1974 *$ & 94 & $2205 * * *$ & 87 \\
\hline \multirow[t]{4}{*}{ Mean plaque area $\left(\mathrm{mm}^{2}\right)$} & $45-49$ & 5.3 & 0.9 & 11.3 & 1.8 & 8.3 & 1.2 & 4.0 & 0.6 & $3.1 * * *$ & 0.7 & $3.6 * * *$ & 0.5 \\
\hline & $50-59$ & 9.9 & 1.8 & 21.0 & 3.2 & 15.5 & 2.2 & $5.3 * *$ & 0.6 & $8.2 * * *$ & 1.5 & $6.8 * * *$ & 0.8 \\
\hline & $55-59$ & 10.6 & 1.7 & 21.7 & 3.2 & 16.1 & 2.1 & 8.3 & 1.1 & $8.8 * *$ & 1.2 & $8.6^{*}$ & 0.9 \\
\hline & $60-65$ & 20.5 & 2.6 & 28.6 & 4.3 & 24.5 & 2.6 & $12.7 * *$ & 1.9 & $15.5 * * *$ & 1.6 & $14.1 * * *$ & 1.4 \\
\hline \multirow[t]{4}{*}{ Maximum plaque area $\left(\mathrm{mm}^{2}\right)$} & $45-49$ & 13.4 & 1.7 & 16.7 & 2.4 & 22.3 & 2.7 & 13.6 & 1.3 & $5.5 * * *$ & 1.1 & $14.6^{* * *}$ & 1.4 \\
\hline & $50-59$ & 20.1 & 2.8 & 28.8 & 4.0 & 36.0 & 4.9 & $11.9 *$ & 0.8 & $12.5 * * *$ & 1.9 & $18.1 * * *$ & 1.7 \\
\hline & $55-59$ & 20.1 & 2.6 & 30.0 & 4.0 & 35.7 & 4.3 & 16.5 & 1.9 & $13.5^{* *}$ & 1.5 & $21.4^{*}$ & 2.0 \\
\hline & $60-65$ & 28.5 & 3.0 & 36.6 & 4.1 & 45.6 & 5.0 & $21.1 * *$ & 2.8 & $21.7 * * *$ & 1.9 & 29.6 *** & 2.8 \\
\hline
\end{tabular}

$S E$ standard error

$P$ value for the comparison between men and women

$* P<0.05, * * P<0.01, * * * P<0.001$

a plaque-based score at either carotid or femoral artery levels was not significantly associated with cardiovascular mortality or myocardial infarction in multivariate analysis, but the femoral plaque score significantly predicted revascularization [16]. The rationale underlying possibly different significance of ATS markers according to different arterial segments is not clear. It has previously been shown that different determinants were accountable for ATS found in different arterial segments in symptomatic and asymptomatic subjects [39, 40]. Hypotheses for such differential patterns include hemodynamic stress related to arterial geometry as well as anatomic, cellular and biochemical variations across different artery locations [41, 42].

In this study, CVRF tended to be more strongly associated with plaque-based markers than with IMT, and this might indirectly suggest that plaque-based markers could outperform IMT in predicting CVD risk. The value of IMT vs. plaque-based markers to predict cardiovascular events has been examined in a few reports with conflicting results. Consistent with our findings, two cross-sectional studies found that plaques were associated with established CVD better than IMT, respectively with ischemic heart disease in a representative sample of older adults [20], and with prevalent CVD in hypertensive individuals [37]. However, the superiority of plaque-based markers over IMT was not consistently found in prospective studies. In the Rotterdam study, which included 3,996 adults with a mean follow-up of 6.1 years, the incidence of stroke was associated more strongly with carotid IMT than a score of carotid plaques [19]. In the same study, carotid IMT and the same carotid plaque score were equally good predictors of incident myocardial infarctions [1]. In contrast, a similar carotid plaque-based score largely outperformed carotid IMT for the prediction of all-cause and cardiovascular mortality in a cohort study of 367 elderly men [22]. In these two prospective studies, the plaque score was quantified by summing the number of segments of the carotid arteries in which a plaque was found, and patients were then grouped in arbitrarily-defined categories. This categorical approach might limit the statistical power of analyses and account for lower prediction of such plaque-based score as compared to IMT in these studies. 
Table 3 Linear regression of main risk factors on different markers of peripheral atherosclerosis at carotid and femoral artery levels

\begin{tabular}{|c|c|c|c|c|c|c|}
\hline & \multicolumn{2}{|l|}{ Carotid } & \multicolumn{2}{|l|}{ Femoral } & \multicolumn{2}{|c|}{ Carotid and femoral } \\
\hline & Coefficient & $\mathrm{P}$ & Coefficient & $\mathrm{P}$ & Coefficient & $\mathrm{P}$ \\
\hline \multicolumn{7}{|l|}{ Mean IMT } \\
\hline Age & 0.28 & $<0.001$ & 0.23 & $<0.001$ & 0.28 & $<0.001$ \\
\hline Sex & 0.06 & ns & -0.14 & 0.002 & -0.11 & 0.010 \\
\hline LDL-cholesterol & 0.11 & 0.013 & 0.14 & 0.001 & 0.15 & $<0.001$ \\
\hline HDL-cholesterol & -0.10 & 0.018 & -0.14 & 0.001 & -0.15 & $<0.001$ \\
\hline Systolic blood pressure & 0.12 & 0.006 & 0.10 & 0.017 & 0.12 & 0.003 \\
\hline Smoking & 0.00 & ns & 0.21 & $<0.001$ & 0.19 & $<0.001$ \\
\hline Diabetes & 0.05 & ns & 0.15 & 0.001 & 0.15 & $<0.001$ \\
\hline \multicolumn{7}{|l|}{ Mean plaque thickness } \\
\hline Age & 0.35 & $<0.001$ & 0.28 & $<0.001$ & 0.37 & $<0.001$ \\
\hline $\operatorname{Sex}$ & -0.10 & 0.019 & -0.17 & $<0.001$ & -0.17 & $<0.001$ \\
\hline LDL-cholesterol & 0.13 & 0.001 & 0.16 & $<0.001$ & 0.18 & $<0.001$ \\
\hline HDL-cholesterol & -0.06 & ns & -0.11 & 0.005 & -0.10 & 0.004 \\
\hline Systolic blood pressure & 0.15 & 0.001 & 0.19 & $<0.001$ & 0.21 & $<0.001$ \\
\hline Smoking & 0.09 & 0.037 & 0.24 & $<0.001$ & 0.21 & $<0.001$ \\
\hline Diabetes & 0.03 & ns & 0.11 & 0.006 & 0.09 & 0.015 \\
\hline \multicolumn{7}{|l|}{ Maximal plaque thickness } \\
\hline Age & 0.30 & $<0.001$ & 0.28 & $<0.001$ & 0.30 & $<0.001$ \\
\hline $\operatorname{Sex}$ & -0.11 & 0.016 & -0.16 & $<0.001$ & -0.20 & $<0.001$ \\
\hline LDL-cholesterol & 0.15 & 0.001 & 0.15 & $<0.001$ & 0.18 & $<0.001$ \\
\hline HDL-cholesterol & -0.02 & ns & -0.10 & 0.008 & -0.03 & ns \\
\hline Systolic blood pressure & 0.15 & 0.001 & 0.18 & $<0.001$ & 0.21 & $<0.001$ \\
\hline Smoking & 0.06 & ns & 0.23 & $<0.001$ & 0.18 & $<0.001$ \\
\hline Diabetes & 0.01 & ns & 0.10 & 0.015 & 0.08 & 0.054 \\
\hline \multicolumn{7}{|l|}{ Mean plaque area } \\
\hline Age & 0.27 & $<0.001$ & 0.19 & $<0.001$ & 0.26 & $<0.001$ \\
\hline Sex & -0.14 & 0.002 & -0.22 & $<0.001$ & -0.22 & $<0.001$ \\
\hline LDL-cholesterol & 0.18 & $<0.001$ & 0.13 & 0.001 & 0.18 & $<0.001$ \\
\hline HDL-cholesterol & -0.08 & 0.044 & -0.10 & 0.008 & -0.12 & 0.002 \\
\hline Systolic blood pressure & 0.13 & 0.003 & 0.17 & $<0.001$ & 0.18 & $<0.001$ \\
\hline Smoking & 0.08 & 0.056 & 0.27 & $<0.001$ & 0.23 & $<0.001$ \\
\hline Diabetes & 0.07 & ns & 0.12 & 0.005 & 0.12 & 0.003 \\
\hline \multicolumn{7}{|l|}{ Maximal plaque area } \\
\hline Age & 0.20 & $<0.001$ & 0.19 & $<0.001$ & 0.19 & $<0.001$ \\
\hline Sex & -0.15 & 0.005 & -0.24 & $<0.001$ & -0.25 & $<0.001$ \\
\hline LDL-cholesterol & 0.19 & $<0.001$ & 0.14 & $<0.001$ & 0.17 & $<0.001$ \\
\hline HDL-cholesterol & -0.08 & 0.107 & -0.10 & 0.009 & -0.06 & 0.226 \\
\hline Systolic blood pressure & 0.15 & 0.002 & 0.16 & $<0.001$ & 0.18 & $<0.001$ \\
\hline Smoking & 0.09 & 0.073 & 0.24 & $<0.001$ & 0.22 & $<0.001$ \\
\hline Diabetes & 0.10 & 0.062 & 0.09 & 0.023 & 0.15 & 0.002 \\
\hline
\end{tabular}

Coefficient normalized beta regression coefficient

To our knowledge, no population-based study has compared the relationship between IMT and continuous plaque-based markers (like thickness or area) with cardiovascular events or conditions. Nevertheless, carotid plaque area has already been compared to carotid IMT in selected populations. Carotid plaque area outperformed IMT for the prediction of cardiovascular events in 152 patients with established coronary artery disease [21], and was more accurate than IMT for defining patients without coronary stenosis on CT angiography in a selected 
Table 4 Variance in predicting markers of peripheral atherosclerosis at carotid and femoral artery levels attributable to age and conventional modifiable risk factors

\begin{tabular}{|c|c|c|c|c|c|c|c|c|c|}
\hline & \multicolumn{3}{|c|}{ Carotid arteries } & \multicolumn{3}{|c|}{ Femoral arteries } & \multicolumn{3}{|c|}{ Carotid and femoral arteries } \\
\hline & Full model & Age and sex & Risk factors & Full model & Age and sex & Risk factors & Full model & Age and sex & Risk factors \\
\hline \multicolumn{10}{|l|}{ Mean IMT } \\
\hline Men & 11.7 & 5.9 & 5.8 & 20.3 & 4.5 & 15.8 & 21.5 & 5.8 & 15.7 \\
\hline Women & 18.3 & 13.4 & 4.9 & 24.5 & 18.2 & 6.3 & 31.6 & 23.7 & 7.9 \\
\hline All & 14.8 & 10.6 & 4.2 & 24.2 & 12.7 & 11.5 & 27.5 & 15.0 & 12.5 \\
\hline \multicolumn{10}{|c|}{ Mean plaque thickness } \\
\hline Men & 23.6 & 18.7 & 4.9 & 26.4 & 8.5 & 17.9 & 36.7 & 19.0 & 17.7 \\
\hline Women & 18.8 & 14.2 & 4.6 & 34.0 & 22.8 & 11.2 & 40.6 & 28.3 & 12.3 \\
\hline All & 22.1 & 17.4 & 4.7 & 33.7 & 19.4 & 14.3 & 41.4 & 27.1 & 14.3 \\
\hline \multicolumn{10}{|c|}{ Maximal plaque thickness } \\
\hline Men & 19.5 & 16.2 & 3.3 & 23.6 & 9.4 & 14.2 & 26.9 & 20.5 & 6.4 \\
\hline Women & 14.2 & 9.3 & 4.9 & 31.7 & 21.3 & 10.4 & 29.6 & 24.9 & 4.7 \\
\hline All & 17.6 & 13.6 & 4.0 & 31.4 & 18.8 & 12.6 & 32.6 & 21.5 & 11.1 \\
\hline \multicolumn{10}{|c|}{ Mean plaque area } \\
\hline Men & 23.8 & 13.1 & 10.7 & 23.5 & 6.1 & 17.4 & 31.8 & 12.0 & 19.8 \\
\hline Women & 14.7 & 10.0 & 4.7 & 29.5 & 16.7 & 12.8 & 34.5 & 20.9 & 13.6 \\
\hline All & 20.4 & 13.2 & 7.2 & 30.1 & 16.6 & 13.5 & 35.9 & 21.0 & 14.9 \\
\hline \multicolumn{10}{|c|}{ Maximal plaque area } \\
\hline Men & 22.8 & 8.7 & 14.1 & 19.8 & 5.1 & 14.7 & 22.8 & 6.6 & 16.2 \\
\hline Women & 7.0 & 4.0 & 3.0 & 28.9 & 17.4 & 11.5 & 22.4 & 11.8 & 10.6 \\
\hline All & 16.5 & 8.0 & 8.5 & 28.4 & 16.7 & 11.7 & 27.8 & 14.6 & 13.2 \\
\hline
\end{tabular}

Full model includes age, sex, LDL-cholesterol. HDL-cholesterol, systolic blood pressure, smoking, and diabetes

Variance for risk factors is estimated by subtracting variance for full model minus variance for age and sex

Table 5 Correlation coefficients between atherosclerosis markers at carotid and femoral artery levels in men and women

\begin{tabular}{|c|c|c|c|c|c|c|c|c|}
\hline & \multicolumn{2}{|l|}{ IMT } & \multicolumn{2}{|c|}{ Mean plaque thickness } & \multicolumn{2}{|c|}{ Maximal plaque thickness } & \multicolumn{2}{|c|}{ Mean plaque area } \\
\hline & Men & Women & Men & Women & Men & Women & Men & Women \\
\hline \multicolumn{9}{|l|}{ Carotid } \\
\hline \multicolumn{9}{|l|}{ IMT } \\
\hline Mean plaque thickness & 0.41 & 0.52 & & & & & & \\
\hline Maximal plaque thickness & 0.35 & 0.46 & 0.95 & 0.95 & & & & \\
\hline Mean plaque area & 0.46 & 0.68 & 0.89 & 0.83 & 0.81 & 0.76 & & \\
\hline Maximal plaque area & 0.42 & 0.72 & 0.83 & 0.73 & 0.76 & 0.71 & 0.96 & 0.97 \\
\hline \multicolumn{9}{|l|}{ Femoral } \\
\hline \multicolumn{9}{|l|}{ MT } \\
\hline Mean plaque thickness & 0.76 & 0.79 & & & & & & \\
\hline Maximal plaque thickness & 0.70 & 0.71 & 0.96 & 0.95 & & & & \\
\hline Mean plaque area & 0.79 & 0.79 & 0.89 & 0.91 & 0.83 & 0.85 & & \\
\hline Maximal plaque area & 0.73 & 0.74 & 0.86 & 0.88 & 0.84 & 0.89 & 0.97 & 0.96 \\
\hline
\end{tabular}

$P<0.001$ for all coefficients

population of patients with known CAD or with moderate to high CV risk. [27].

Differences in the relationships between different ATS markers and conventional CVRF have already been found by others [43], and can be explained by methodological and physiological factors. From a physiological perspective, IMT is influenced by both intima and non-intima related processes. Examples include hypertensive medial hypertrophy [12] or adaptive responses of IMT to factors not related to ATS such as shear stress [11]. By contrast, plaques are more specifically 
related to the ATS process. Methodologically, the measurement of plaque-based markers - as compared to IMT-assesses a frankly pathological process. From a screening perspective, IMT and plaque-based markers might act as synergistic rather than redundant approaches [3]. Like Al-Shali et al. [43], we found a substantially lower correlation between IMT and plaque-based ATS markers than between the different plaque-based markers, which suggests that IMT and plaque-based markers may convey different information. As IMT and plaque-based markers not only differ in their relationship with the traditional CVRF but also seem to have different genetic determinants, it has been suggested to consider them as completely different entities [44]. Thus, the use of IMT or plaque-based markers as proxy measurements of atherosclerosis may lead to different conclusions and might have a complementary role. For example, while carotid IMT seems to better predict stroke than plaque-based markers [19], the use of both types of markers may further improve risk prediction [24]. Also, IMT may be the only marker usable in individuals without plaques, particularly at an early stage of ATS development [45]. Similarly, our finding that the correlations between the same ATS markers at carotid and femoral levels were only fair (correlation coefficients $<0.5$ ) suggest that extending measurements of any ATS marker to both the carotid and femoral levels-as opposed to screening in only one artery level- might improve CVD risk prediction.

Some consistency in the high correlation between all plaque-based markers can be expected as all these markers intercorrelate for morphological reasons: e.g. maximum thickness is a component of mean thickness and plaque thickness is a component of plaque area. As mean plaque area is based on all visualized lesions in the considered artery segments, and not only on the thickest one, one would expect that this indicator provides the most comprehensive available measurement of both ATS and life-long exposure to risk factors. However, our findings did not demonstrate a clear superiority of the areabased markers as compared to thickness-based markers, although area-based markers tended to display the highest variance explained by modifiable risk factors (carotid score and scores based on carotid and femoral arteries). Of interest, no other study has yet examined the relationship between plaque area at femoral level and outcomes (whether CVRF, established CV disease or incident CV events). In our study, plaque area was also the least reproducible of the ATS markers. This is anticipated in view of increased error in assessing two dimensions (area) vs. one dimension (plaque thickness). In the future, three-dimensional assessment of plaque volume might provide an advantage compared to markers based on area or one-dimension measurements especially when ad hoc software allowing automatic or semiautomatic measurement becomes available [18]. Pending such technological advances, it is easier and quicker to measure plaque thickness than plaque area, so that a possible slight advantage of plaque area in terms of prediction of cardiovascular risk might not be worth the longer time and greater expertise needed for measurement of area versus thickness.

Our study has several limitations. Its cross-sectional design does not allow causal inference in the relationships between CVRF and ATS markers. The relatively small sample size did not allow to confirm our findings in subgroups of participants at intermediate CVD risk, who are likely to benefit most of screening with such markers for better risk stratification [46]. Furthermore, the assessment of the relationship between ATS markers and CVRF can only suggest a potential role of these markers to predict cardiovascular risk and need to be confirmed in prospective studies using hard cardiovascular outcomes. Also, the predominantly African descent of this population may limit the generalization of the results to other ethnicities. On the other hand, the population-based design and high participation rate are strengths of our study.

In conclusion, while ultrasound detection of subclinical peripheral atherosclerosis appears to be a promising tool for the non-invasive identification of subjects at high risk for cardiovascular disease, there is still ample debate on which markers and which artery sites can best predict cardiovascular outcomes. By providing a head to head comparison of IMT, plaque thickness and plaque area in relation to CVRF at both the carotid and femoral artery levels, this study suggests that plaque-based ATS markers, on one hand, and femoral location, on the other hand, might play an important role in the prediction of the cardiovascular risk and should be examined in addition to IMT or other markers assessed at the sole carotid level in future prospective studies with hard CVD outcomes.

Acknowledgments The authors thank all the survey officers and the Ministry of Health, Republic of Seychelles, for continued support to epidemiological research. The Seychelles Heart Study III was conducted by the Ministry of Health of the Republic of Seychelles, with the collaboration and support of the University Institute of Social and Preventive of Lausanne (Switzerland); the Canton Laboratory of Hematology and Clinical Chemistry, St-Gallen (Switzerland); and the World Health Organization (WHO). The software for semi-automatic measurement of IMT (M'ATH v 2.1) was provided by Metris, 95100 Argenteuil, France.

Conflict of interest None.

\section{References}

1. van der Meer I, Bots M, Hofman A, Iglesias del Sol A, van der Kuip D, Witteman J (2004) Predictive value of noninvasive measures of atherosclerosis for incident myocardial infraction. The Rotterdam study. Circulation 109:1089-1094

2. Lorenz M, Markus HS, Bots ML, Rosvall M, Sitzer M (2007) Prediction of clinical cardiovascular events with carotid intima-media 
thickness: a systematic review and meta-analysis. Circulation 115:459-467

3. Kullo IJ, Malik AR (2007) Arterial ultrasonography and tonometry as adjuncts to cardiovascular risk stratification. J Am Coll Cardiol 49:1413-1426

4. Naghavi M, Falk E, Hecht H, Jamieson M, Kaul S, Berman D, Fayad Z, Budoff M, Rumberger J, Naqvi T, Shaw L, Faegermann O, Cohn J, Bahr R, Koenig W, Demirivic J, Arking D, Herrera V, Badimon J, Goldstein J, Rudy Y, airaksinen J, Schwartz R, Riley W, Mendes R, Douglas P, Shah P, for the SHAPE Task Force. From vulnerable plaque to vulnerable patient-part III: executive summary of the screening for heart attack prevention and education (SHAPE) task force report. Am J Cardiol 2006; 98 [suppl]: $2 \mathrm{H}-15 \mathrm{H}$

5. De Groot E, Hovingh G, Wiegman A, Duriez P, Smit A, Fruchart JC, Kastelein J. Measurement of arterial wall thickness as a surrogate marker for atherosclerosis. Circulation 2004; 109 [Suppl 3]: III-33-III-38

6. Bots M, Hoes A, Koudstaal P, Hofman A, Grobee D (1997) Common carotid intima-media thickness and risk of stroke and myocardial infarction. The rotterdam study. Circulation 96:1432-1437

7. Chambless L, Heiss G, Folsom A, Rosamond W, Szklo M, Sharrett A, Clegg L (1997) Association of coronary heart disease incidence with carotid arterial wall thickness and major risk factors: the atherosclerosis risk in communities (ARIC) study, 1987-93. Am J Epidemiol 146:483-494

8. Hodis H, Mack W, LaBree L, Selzer R, Liu C-R, Liu C-H, Azen $S$ (1998) The role of carotid arterial intima-media thickness in predicting clinical coronary events. Ann Intern Med 128:262-269

9. O'leary D, Polak J, Kronmal R, Manolio T, Burke G, Wolfson S, For the cardiovascular health study collaborative research group (1999) Carotid-artery intima and media thickness as a risk factor for myocardial infarction and stroke in older adults. N Engl J Med 340:14-22

10. Taylor A, Merz C, Udelson J (2003) 34th bethesda conference: can atherosclerosis imaging techniques improve the detection of patients at risk for ischemic heart disease? J Am Coll Cardiol 41:1855-1858

11. Bots M, Hofman A, Grobbee DE (1997) Increased common carotid intima-media thickness. Adaptive response or a reflection of atherosclerosis? findings from the rotterdam study. Stroke 28:2442-2447

12. Roman M, Pickering T, Pini R, Schwartz J, Devereux R (1995) Prevalence and determinants of cardiac and vascular hypertrophy in hypertension. Hypertension 26:369-373

13. Iglesias del Sol A, Moons K, Hollander M, Hofman A, Koudstaal P, Grobbee D, Breteler M, Witteman J, Bots M (2001) Is carotid intima-media thickness useful in cardiovascular disease risk assessment? Stroke 32:1532-1538

14. Salonen J, Salonen R (1991) Ultrasonographically assessed carotid morphology and the risk of coronary heart disease. Arterioscler Thromb 11:1245-1249

15. Belcaro G, Nicolaides A, Ramaswami G, Cesarone M, De Sanctis M, Incandela L, Ferrari P, Geroulakos G, Barsotti A, Griffin M, Dhanjil S, Sabetai M, Bucci M, Martines G (2001) Carotid and femoral ultrasound morphology screening and cardiovascular events in low risk subjects: a 10-year follow-up study. (The CAFES-CAVE study). Atherosclerosis 156:379-387

16. Held C, Hjemdahl P, Eriksson S, Björkander I, Forslund L, Rehnqvist N (2001) Prognostic implications of intima-media thickness and plaques in the carotid and femoral arteries in patients with stable angina pectoris. Eur Heart J 22:62-72

17. Hollander M, Bots M, Iglesias del Sol A, Koudstaal P, Witteman J, Grobbee D, Hofman A, Breteler M (2002) Carotid plaques increase the risk of stroke and subtypes of cerebral infarction in asymptomatic elderly. The rotterdam study. Circulation 105: 2872-2877

18. Spence JD (2002) Ultrasound measurement of carotid plaque as a surrogate outcome for coronary artery disease. Am J Cardiol 89:10B-16B

19. Hollander M, Hak AE, Koudstaal PJ, Bots M, Grobee D, Hofman A, Witteman J, Breteler MB (2003) Comparison between measures of atherosclerosis and risk of stroke. The rotterdam study. Stroke 34:2367-2373

20. Tartière JM, Henry O, Safar H, Bureau JM, Girerd X, Safar M, Blacher J (2003) Carotid intima-media thickness and carotid and/ or iliofemoral plaques: comparison of two markers of cardiovascular risk in hypertensive patients. J Hypertens 21:739-746

21. Chan S, Mancini G, Kuramoto L, Schulzer M, Frohlich J, Ignaszewski A (2003) The prognostic importance of endothelial dysfunction and carotid atheroma burden in patients with coronary artery disease. J Am Coll Cardiol 42:1037-1043

22. Störk S, van den Beld A, von Schacky C, Angermann C, Lamberts S, Grobbee D, Bots M (2004) Carotid plaque burden, stiffness and mortality risk in elderly men. A prospective, population-based cohort study. Circulation 110:344-348

23. Schmidt C, Fagerberg B, Hulthe J (2005) Non-stenotic echolucent ultarsound-assessed femoral artery plaques are predictive for future cardiovascular events in middle-aged men. Atherosclerosis 181:125-130

24. Rosvall M, Janzon L, Berglund G, Engström G, Hedblad B (2005) Incidence of stroke is related to carotid IMT even in the absence of plaque. Atherosclerosis 179:325-331

25. Baldassarre D, Amato M, Pustina L, Castelnuovo S, Sanvito S, Gerosa L, Veglia F, Keidar S, Tremoli E, Sirtori C (2007) Measurement of carotid artery intima-media thickness in dyslipidemic patients increases the power of traditional risk factors to predict cardiovascular events. Atherosclerosis 191:403-408

26. Spence J, Eliasziw M, DiCicco M, Hackam D, Galil R, Lohmann T (2002) Carotid plaque area. A tool for targeting and evaluating vascular preventive therapy. Stroke 33:2916-2922

27. Brook R, Bard R, Patel S, Rubenfire M, Clarke N, Kazerooni E, Wakefield T, Henke P, Eagle K (2006) A negative carotid plaque area test is superior to other non-invasive atherosclerosis studies for reducing the likelihood of having underlying significant coronary artery disease. Arterioscler Thromb Vasc Biol 26:656-662

28. Management and information system division (2004) Statistical abstracts. Victoria, Seychelles

29. Bovet P, Shamlaye C, Kitua A, Riesen WF, Paccaud F, Darioli R (1991) High prevalence of cardiovascular risk factors in the Seychelles (Indian Ocean). Arterioscler Thromb 11:1730-1736

30. Bovet P, Shamlaye C, Gabriel A, Riesen W, Paccaud F (2006) Prevalence of cardiovascular risk factors in a middle-income country and estimated cost of a treatment strategy. BMC Public health 6:9

31. Bovet P, Romain S, Shamlaye C, Mendis S, Darioli R, Riesen W, Tappy L, Paccaud F (2009) Divergent 15-year trend in traditional and metabolic risk factors of cardiovascular diseases in the seychelles. Cardiovasc Diabetol 8:34

32. Perret F, Bovet P, Shamlaye C, Paccaud F, Kappenberger L (2000) High prevalence of peripheral atherosclerosis in a rapidly developing country. Atherosclerosis 153:9-21

33. Rodondi N, Yerly P, Gabriel A, Riesen W, Burnier M, Paccaud F, Bovet P (2007) Microalbuminuria, but not cystatin C, is associated with carotid atherosclerosis in middle-aged adults. Nephrol Dial Transplant 22:1107-1114

34. American Diabetes Association (2004) Diagnosis and classification of diabetes mellitus. Diabetes Care 27:S5-S10

35. Touboul PJ, Hennerici MG, Meairs S, Adams H, Amarenco P, Desvarieux M, Ebrahim S, Fatar M, Hernandez R, Kownator S, Prati P, Rundek T, Taylor A, Bronstein N, Csiba L, Vicaut E, 
Woo K, Zannad F (2004) Mannheim intima-media thickness consensus. Cerebrovasc Dis 18:346-349

36. Depairon M, Chessex S, Sudre P, Rodondi N, Doser N, Chave JP, Riesen W, Nicod P, Darioli R, Telenti A, Mooser V (2001) With the Swiss HIV cohort study. Premature atherosclerosis in HIVinfected individuals - focus on protease inhibitor therapy. Aids 15:329-334

37. Ebrahim S, Papacosta O, Whincup P, Wannamethee G, Walker M, Nicolaides A, Dhanjil S, Griffin M, Belcaro G, Rumley A, Lowe G (1999) Carotid plaque, intima-media thickness, cardiovascular risk factors, and prevalent cardiovascular disease in men and women; the British regional heart study. Stroke 30:841-850

38. Tang R, Hennig M, Bond MG, Hollweck R, Mancia G, Zanchetti A (2005) Quality control of B-mode ultrasonographic measurement of carotid intima-media thickness: the European lacidipine study on atherosclerosis. J Hypertens 23:1047-1054

39. Van der Meer I, del Sol A, Hak E, Bots M, Hofman A, Wittman J (2003) Risk factors for progression of atherosclerosis measured at multiple sites in the arterial tree: the rotterdam study. Stroke 34:2374-2379

40. Diehm N, Shang A, Silvestro A, Do D, Dick F, Schmidli J, Mahler F, Baumgartner I (2006) Association of cardiovascular risk factors with pattern of lower limb atherosclerosis in 2659 patients undergoing angioplasty. Eur J Endovasc Surg 31:59-63
41. Solberg L, Eggen D (1971) Localisation and sequence of development of atherosclerotic lesions in the carotid and vertebral arteries. Circulation 43:711-724

42. McGill H, McMahan C, Herderick E, Tracy R, Malcom G, Zieske A (2000) Effects of coronary heart disease risk factors on atherosclerosis of selected regions of the aorta and right coronary artery. PDAY research group. Arterioscler Thromb Vasc Biol 20:836-845

43. Al-Shali K, House A, Hanley A, Khan H, Harris S, Mamakeesick M, Zimman B, Fenster A, Spence J, Hegele R (2005) Differences between carotid wall morphological phenotypes measured by ultrasound in one, two, and three dimensions. Atherosclerosis 178: 319-325

44. Spence JD (2006) Technology insight: ultrasound measurement of carotid plaque - patient management, genetic research, and therapy evaluation. Nat Clin Pract Neurol 2(11):611-619

45. Bonithon-Kopp C, Touboul PJ, Berr C, Leroux C, Mainard F, Courbon D, Ducimetiere P (1996) Relation of intima-media thickness to atherosclerotic plaques in carotid arteries. The vascular aging (EVA) study. Arterioscler Thromb Vasc Biol 16:310-316

46. Greenland P, Smith S, Grundy S (2001) Improving coronary heart disease risk assessment in asymptomatic people. Role of traditional risk factors and non-invasive cardiovascular tests. Circulation 104:1863-1867 